\title{
INHERITED PROPERTIES OF EFFECT ALGEBRAS PRESERVED BY ISOMORPHISMS
}

\author{
JAN PASEKA $^{a, *}$, ZDENKA RIEČANOVÁ ${ }^{b}$ \\ a Department of Mathematics and Statistics, Faculty of Science, Masaryk University, Kotlářská 2, \\ CZ-611 37 Brno, Czech Republic \\ ${ }^{b}$ Department of Mathematics, Faculty of Electrical Engineering and Information Technology, Slovak University \\ of Technology, Ilkovičova 3, SK-812 19 Bratislava, Slovak Republic \\ * corresponding author: paseka@math.muni.cz
}

ABSTRACT. We show that isomorphism of effect algebras preserves properties of effect algebras derived from effect algebraic sum $\oplus$ of elements. These are partial order, order convergence, order topology, existence of states and other important properties. However, there are properties of effect algebras for which the preservation of the $\oplus$-operation is not substantial and they need not be preserved.

KEYWORDS: effect algebras; operator effect algebras; isomorphisms; operator representations of effect algebras..

AMS Mathematics Subject Classification: 06C15, (03G12, 81P10).

\section{INTRODUCTION}

In the quantum-mechanical framework, the elements of an effect algebra represent quantum effects, meaning elementary yes-no measurements that may be unsharp. The standard Hilbert space effect algebra $\mathcal{E}(\mathcal{H})$ on a complex Hilbert space $\mathcal{H}$ is the set $\mathcal{E}(\mathcal{H})$ of all positive operators dominated by the identity operator $I$ on $\mathcal{H}$. So called interval effect algebras form a further important class of effect algebras. These are effect algebras possessing an ordering set of states, which is equivalent to the condition that these effect algebras can be represented by positive linear operators densely defined in an infinite-dimensional complex Hilbert space $\mathcal{H}$ (see [18]). Here, by the operator representation of effect algebras (initiated by questions of M. Znojil at the 9th PHHQP workshop in Hangzhou, China) we mean their isomorphism with sub-effect algebras of the standard Hilbert space effect algebra $\mathcal{E}(\mathcal{H})$ on the complex Hilbert space $\mathcal{H}$.

In this paper we show that isomorphisms of effect algebras inherit the partial order on them, and consequently also the order convergence on them and other important properties. However, we also show examples of properties that need not be inherited by isomorphisms of effect algebras (e.g., sequential product of elements).

\section{BASIC DEFINITIONS AND SOME KNOWN FACTS}

\subsection{EfFect AlgEbras AND genERAlized EFFECT ALGEBRAS}

Definition 2.1. 3 A partial algebra $(E ; \oplus, 0,1)$ is called an effect algebra if 0,1 are two distinguished elements and $\oplus$ is a partially defined binary operation on $E$ which satisfies the following conditions for any $x, y, z \in E$ :

(E1) $x \oplus y=y \oplus x$ if $x \oplus y$ is defined,

(E2) $(x \oplus y) \oplus z=x \oplus(y \oplus z)$ if one side is defined,

(E3) for every $x \in E$ there exists a unique $y \in E$ such that $x \oplus y=1$ (we put $x^{\prime}=y$ and say that $x^{\prime}$ is a supplement of $x$ ),

(E4) If $1 \oplus x$ is defined then $x=0$.

We often denote the effect algebra $(E ; \oplus, 0,1)$ briefly by $E$. On every effect algebra $E$ the partial order $\leq$, binary relation $\perp$ and partial binary operation $\ominus$ can be introduced as follows: $x \leq y$ and $x \perp z$ and $y \ominus x=z$ iff $x \oplus z$ is defined and $x \oplus z=y$.

Generalizations of effect algebras (i.e. without a top element 1 ) have been introduced and studied in [3], [5], 6] and [9].

Definition 2.2. (1.) A generalized effect algebra ( $E$, $\oplus, 0)$ is a set $E$ with an element $0 \in E$ and a partial binary operation $\oplus$ satisfying for any $x, y, z \in E$ the conditions

(GE1) $x \oplus y=y \oplus x$ if one side is defined,

(GE2) $(x \oplus y) \oplus z=x \oplus(y \oplus z)$ if one side is defined,

(GE3) if $x \oplus y=x \oplus z$ then $y=z$,

(GE4) if $x \oplus y=0$ then $x=y=0$,

(GE5) $x \oplus 0=x$ for all $x \in E$.

(2.) Define a binary relation $\leq$ on $E$ by

$$
x \leq y \text { iff for some } z \in E, x \oplus z=y .
$$

The significant property of a generalized effect algebra $(E ; \oplus, 0)$ is that every interval $[0, q]$, for $q \in E$, $q \neq 0$, is an effect algebra with $\oplus$ restricted to $[0, q]$. 
Every effect algebra $E$ is also a generalized effect algebra and a generalized effect algebra is also an effect algebra iff it includes the top element.

Definition 2.3. A nonempty subset $Q$ of an effect algebra (generalized effect algebra) $E$ is called a $s u b$ effect algebra (sub-generalized effect algebra) of $E$ iff:

(1.) if at least two of the elements $x, y, z \in E$ with $x \oplus y=z$ are in $Q$ then all $x, y, z$ are in $Q$;

(2.) $1 \in Q$ when $E$ is an effect algebra.

We say that a finite system $F=\left(x_{k}\right)_{k=1}^{n}$ of not necessarily different elements of an effect algebra $(E ; \oplus, 0,1)$ is orthogonal if $x_{1} \oplus x_{2} \oplus \cdots \oplus x_{n}$ (written $\bigoplus_{k=1}^{n} x_{k}$ or $\left.\bigoplus F\right)$ exists in $E$. Here we define $x_{1} \oplus x_{2} \oplus \cdots \oplus x_{n}=\left(x_{1} \oplus x_{2} \oplus \cdots \oplus x_{n-1}\right) \oplus x_{n}$ supposing that $\bigoplus_{k=1}^{n-1} x_{k}$ is defined and $\bigoplus_{k=1}^{n-1} x_{k} \leq x_{n}^{\prime}$. We also define $\bigoplus \varnothing=0$. An arbitrary system $G=\left(x_{\kappa}\right)_{\kappa \in H}$ of not necessarily different elements of $E$ is called orthogonal if $\bigoplus K$ exists for every finite $K \subseteq G$. We say that for an orthogonal system $G=\left(x_{\kappa}\right)_{\kappa \in H}$ the element $\bigoplus G$ (more precisely $\bigoplus_{E} G$ ) exists iff $\bigvee\{\bigoplus K \mid K \subseteq G$ is finite $\}$ exists in $E$, and then we put $\bigoplus G=\bigvee\{\bigoplus K \mid K \subseteq G$ is finite $\}$. (Here we write $G_{1} \subseteq G$ iff there is $H_{1} \subseteq H$ such that $\left.G_{1}=\left(x_{\kappa}\right)_{\kappa \in H_{1}}\right)$.

\subsection{TOPOLOGIES ON ORDERED SETS}

Definition 2.4. (1.) A preordered set $(\Lambda ; \leq)$ is called a directed (upwards) set of indices if the following conditions are satisfied:
(a) $\alpha \leq \alpha$,
(b) $\alpha \leq \beta, \beta \leq \gamma$ implies $\alpha \leq \gamma$,
(c) for all $\alpha, \beta \in \Lambda$ there exists $\gamma \in \Lambda$ such that $\alpha, \beta \leq \gamma$.

A net $\left(a_{\alpha}\right)_{\alpha \in \Lambda}$ is a family of not necessary different elements which have indices from a directed set of indices $\Lambda$.

(2.) A net $\left(a_{\alpha}\right)_{\alpha \in \Lambda}$ of elements of a poset $(P ; \leq)$ is increasingly directed if $a_{\alpha} \leq a_{\beta}$ for all $\alpha, \beta \in \Lambda$ such that $\alpha \leq \beta$, and then we write $a_{\alpha} \uparrow$. If moreover $a=\bigvee\left\{a_{\alpha} \mid \alpha \in \Lambda\right\}$ we write $a_{\alpha} \uparrow a$ and we call such a net increasing to $a$. The meaning of $a_{\alpha} \downarrow$ and $a_{\alpha} \downarrow a$ is dual (decreasingly directed or filtered).

(3.) A net $\left(a_{\alpha}\right)_{\alpha \in \Lambda}$ of elements of a poset $(P ; \leq)$ order converges ( $(o)$-converges, for short) to a point $a \in P$ if there are nets $\left(u_{\alpha}\right)_{\alpha \in \Lambda}$ and $\left(v_{\alpha}\right)_{\alpha \in \Lambda}$ of elements of $P$ such that

$$
a \uparrow u_{\alpha} \leq a_{\alpha} \leq v_{\alpha} \downarrow a .
$$

We write $a_{\alpha} \stackrel{(o)}{\longrightarrow} a$ in $P$ (or briefly $\left.a_{\alpha} \stackrel{(o)}{\longrightarrow} a\right)$.

Definition 2.5. The order topology (denoted by $\tau_{0}^{P}$ or shortly $\left.\tau_{0}\right)$ on a poset $(P ; \leq)$ is the finest (strongest) topology on $P$ such that for every net $\left(a_{\alpha}\right)_{\alpha \in \Lambda}$ of elements of $P$,

$$
a_{\alpha} \stackrel{(o)}{\longrightarrow} a \text { in } P \Longrightarrow a_{\alpha} \stackrel{\tau_{0}^{P}}{\longrightarrow} a,
$$

where $a_{\alpha} \stackrel{\tau_{0}^{P}}{\longrightarrow} a$ denotes that $\left(a_{\alpha}\right)_{\alpha \in \Lambda}$ converges to $a \in P$ in the topological space $\left(P, \tau_{0}^{P}\right)$.

Clearly, $a_{\alpha} \uparrow a \Rightarrow a_{\alpha} \stackrel{(o)}{\longrightarrow} a$ because $a \uparrow a_{\alpha} \leq a_{\alpha} \leq$ $a \downarrow a$ and $a_{\alpha} \downarrow a \Rightarrow a_{\alpha} \stackrel{(o)}{\longrightarrow} a$, because $a \uparrow a \leq a_{\alpha} \leq$ $a_{\alpha} \downarrow a$ (see [7], [8], [12], [13]).

Theorem 2.6 ([11, Theorem 2.1.21]). Let $(P, \leq)$ be a poset and $F \subseteq P$. Then $F$ is $\tau_{0}$-closed iff for every net $\left(a_{\alpha}\right)_{\alpha \in \Lambda}$ of elements of $P$,

(CS) $\left(a_{\alpha} \in F, \alpha \in \Lambda, a_{\alpha} \stackrel{(o)}{\longrightarrow} a\right) \Rightarrow x \in F$.

\subsection{Morphisms, EMBEDdings AND ISOMORPHISMS OF EFFECT ALGEBRAS}

Recall the following definitions, needed in what follows.

Definition $2.7([2,18])$. Let $\left(E_{1} ; \oplus_{1}, 0_{1}, 1_{1}\right),\left(E_{2}\right.$; $\left.\oplus_{2}, 0_{2}, 1_{2}\right)$ be effect algebras. A mapping $\varphi: E_{1} \rightarrow E_{2}$ is called

(1.) a morphism, if

(a) $\varphi\left(0_{1}\right)=0_{2}, \varphi\left(1_{1}\right)=1_{2}$,

(b) for all $a, b \in E_{1}$ : if $a \oplus_{1} b$ exists then $\varphi(a) \oplus_{2} \varphi(b)$ exists, in which case $\varphi\left(a \oplus_{1} b\right)=\varphi(a) \oplus_{2} \varphi(b)$,

(2.) an ordering morphism, if it is a morphism and, for all $a, b \in E_{1}, a \leq_{1} b$ iff $\varphi(a) \leq_{2} \varphi(b)$,

(3.) an embedding (also called a monomorphism), if $\varphi$ is injective and

(a) $\varphi\left(0_{1}\right)=0_{2}, \varphi\left(1_{1}\right)=1_{2}$,

(c) for all $a, b \in E_{1}: a \oplus_{1} b$ exists iff $\varphi(a) \oplus_{2} \varphi(b)$ exists, in which case $\varphi\left(a \oplus_{1} b\right)=\varphi(a) \oplus_{2} \varphi(b)$,

(4.) an isomorphism, if $\varphi$ is bijective embedding,

(5.) a positive operator valued state (POVS for short) on $E_{1}$ iff $\varphi$ is a morphism into $E_{2}=\mathcal{E}(\mathcal{H})$ for some complex Hilbert space $\mathcal{H}$,

(6.) a Hilbert space effect-representation of $E_{1}$ iff $\varphi$ is an embedding into $E_{2}=\mathcal{E}(\mathcal{H})$ for some complex Hilbert space $\mathcal{H}$.

Clearly, every embedding $\varphi$ is an isomorphism of effect algebras $E_{1}$ and $\varphi\left(E_{1}\right) ; \varphi\left(E_{1}\right)$ is a sub-effect algebra of $E_{2}$; and a composition of morphisms (embeddings, isomorphisms) is again a morphism (embedding, isomorphism). Every morphism of effect algebras preserves supplements.

Recall that $\varphi$ is an isomorphism of effect algebras iff $\varphi$ is bijective and both $\varphi$ and $\varphi^{-1}$ are morphisms of effect algebras.

Lemma 2.8. Let $\left(E_{1} ; \oplus_{1}, 0_{1}, 1_{1}\right)$ and $\left(E_{2} ; \oplus_{2}, 0_{2}, 1_{2}\right)$ be effect algebras and let $\varphi: E_{1} \rightarrow E_{2}$ be a morphism of effect algebras. Then $\varphi$ is order-preserving 
and, for any orthogonal system $G=\left(x_{\kappa}\right)_{\kappa \in H}$ of not necessarily different elements of $E_{1}$, the system $\varphi(G)=\left(\varphi\left(x_{\kappa}\right)\right)_{\kappa \in H}$ is again orthogonal.

Proof. Assume that $a, b \in E_{1}, a \leq_{1} b$. Then there is an element $c \in E_{1}$ such that $a \oplus_{1} c=b$. It follows that $\varphi(b)=\varphi\left(a \oplus_{1} c\right)=\varphi(a) \oplus_{2} \varphi(c) \geq_{2} \varphi(a)$.

Now, let $L \subseteq \varphi(G)$ be finite. Then there is a finite subset $F \subseteq H$ such that $L=\left(\varphi\left(x_{\kappa}\right)\right)_{\kappa \in F}$. Put $K=$ $\left(x_{\kappa}\right)_{\kappa \in F}$. Then $\bigoplus_{E_{1}} K$ exists and hence $\bigoplus_{E_{2}} L$ exists and $\bigoplus_{E_{2}} L=\varphi\left(\bigoplus_{E_{1}} K\right)$. It follows that $\varphi(G)=$ $\left(\varphi\left(x_{\kappa}\right)\right)_{\kappa \in H}$ is orthogonal.

Proposition 2.9. Let $\left(E_{1} ; \oplus_{1}, 0_{1}, 1_{1}\right)$ and $\left(E_{2} ; \oplus_{2}\right.$, $\left.0_{2}, 1_{2}\right)$ be effect algebras and let $\varphi: E_{1} \rightarrow E_{2}$ be a morphism of effect algebras. Then the following conditions are equivalent:

(1.) $\varphi$ is an ordering morphism.

(2.) $\varphi$ is an embedding.

Proof. Assume that $a, b \in E_{1}$. Then $a \leq_{1} b^{\prime}$ iff $a \oplus_{1} b$ exists and $\varphi(a) \leq_{1} \varphi\left(b^{\prime}\right)$ iff $\varphi(a) \leq_{1} \varphi(b)^{\prime}$ iff $\varphi(a) \oplus_{1}$ $\varphi(b)$ exists. Hence $\varphi$ is an ordering morphism iff $\varphi$ is an embedding.

Theorem 2.10. Let $(E ; \oplus, 0,1)$ be an effect algebra and let $\mathcal{H}$ be some complex Hilbert space. For a map $\varphi: E \rightarrow \mathcal{E}(\mathcal{H})$ the following conditions are equivalent:

(1.) $\varphi$ is an ordering positive operator valued state.

(2.) $\varphi$ is an embedding.

(3.) $\varphi$ is a Hilbert space effect-representation of $E$ in $\mathcal{H}$.

Proof. The equivalence between (1.) and (2.) follows from Proposition 2.9 the equivalence between (2.) and (3.) follows from Definition 2.7. (2.), (5.) and (6.).

Definition $2.11([2,[14,[1])$. (1.) A map $\omega: E$ $\rightarrow[0,1] \subseteq \mathbb{R}$ is a state on an effect algebra $E$ if $\omega(0)=0, \omega(1)=1$ and $\omega(x \oplus y)=\omega(x)+\omega(y)$ whenever $x \leq y^{\prime}, x, y \in E$.

(2.) A set $\mathcal{M}$ of states on an effect algebra $E$ is called an ordering set of states if for any $a, b \in E$ the condition $a \leq b$ iff $\omega(a) \leq \omega(b)$ for all $\omega \in \mathcal{M}$, is satisfied.

(3.) A state $\omega$ on an effect algebra $E$ is called $\sigma$ additive if, for every countable net $\left(x_{n}\right)_{n \in \mathbb{N}}$ of elements of $E, x_{n} \uparrow x \Longrightarrow \omega\left(x_{n}\right) \rightarrow \omega(x)$.

(4.) A state $\omega$ on an effect algebra $E$ is called (o)continuous (order-continuous) if, for every net $\left(x_{\alpha}\right)_{\alpha \in \lambda}$ of elements of $E, x_{\alpha} \stackrel{(o)}{\longrightarrow} x$ implies $\omega\left(x_{\alpha}\right) \rightarrow$ $\omega(x)$ (equivalently $x_{\alpha} \uparrow x$ implies $\omega\left(x_{\alpha}\right) \uparrow \omega(x)$ ).

(5.) A state $\omega$ on an effect algebra $E$ is called completely additive if for any orthogonal system $\left(x_{\kappa}\right)_{\kappa \in H}$ of not necessarily different elements of $E$ such that $\bigoplus\left\{x_{\kappa} \mid \kappa \in H\right\}$ exists, $\omega\left(\bigoplus\left\{x_{\kappa} \mid\right.\right.$ $\kappa \in H\})=\sum\left\{\omega\left(x_{\kappa}\right) \mid \kappa \in H\right\}=\sup \left\{\sum\left\{\omega\left(x_{\kappa}\right) \mid\right.\right.$ $\kappa \in F\} \mid F \subseteq H, F$ finite set $\}$.

It follows that states on effect algebras are exactly morphisms from them into $[0,1]$. Note that any $(o)$ continuous state is completely additive and also any completely additive state is $\sigma$-additive.

Moreover, it was proved in [18] that, for an effect algebra $E$, there exists a complex Hilbert space $\mathcal{H}$ such that $E$ has a Hilbert space effect-representation into $\mathcal{E}(\mathcal{H})=[0, I]_{\mathcal{B}^{+}(\mathcal{H})}$, where $\mathcal{B}^{+}(\mathcal{H})$ are positive bounded operators on $\mathcal{H}$ iff there exists an ordering set $\mathcal{M}$ of states on $E$ and then $\mathcal{H}=l_{2}(\mathcal{M})$.

\section{BASIC PROPERTIES OF ISOMORPHISMS OF EFFECT ALGEBRAS AND OPERATOR REPRESENTATIONS}

Roughly speaking, the operator representations of abstract effect algebras (if they exist) are their isomorphisms with operator effect algebras in some complex Hilbert space $\mathcal{H}$. More precisely, they are their isomorphisms with sub-effect algebras of the standard Hilbert space effect algebra $\mathcal{E}(\mathcal{H})$. In such a case it may be interesting to know which properties of the initial effect algebras are inherited for those isomorphic operator effect algebras.

Let us start our considerations with properties of two isomorphic abstract effect algebras.

Theorem 3.1. Let $\left(E_{1} ; \oplus_{1}, 0_{1}, 1_{1}\right)$ and $\left(E_{2} ; \oplus_{2}\right.$, $\left.0_{2}, 1_{2}\right)$ be effect algebras and let $\varphi: E_{1} \rightarrow E_{2}$ be an isomorphism of effect algebras. Then

(1.) For all $a, b \in E_{1}, a \leq_{1} b$ if and only if $\varphi(a) \leq_{2}$ $\varphi(b)$.

(2.) For all $S \subseteq E_{1}, \bigvee_{E_{1}} S$ exists if and only if $\bigvee_{E_{2}} \varphi(S)$ exists, in which case $\bigvee_{E_{2}} \varphi(S)=$ $\varphi\left(\bigvee_{E_{1}} S\right)$.

(3.) For any increasingly directed net $\left(a_{\alpha}\right)_{\alpha \in \Lambda}$ of elements of $E_{1}$ and $a \in E_{1}, a_{\alpha} \uparrow a$ if and only if $\varphi\left(a_{\alpha}\right) \uparrow \varphi(a)$.

(4.) For any decreasingly directed net $\left(a_{\alpha}\right)_{\alpha \in \Lambda}$ of elements of $E_{1}$ and $a \in E_{1}, a_{\alpha} \downarrow a$ if and only if $\varphi\left(a_{\alpha}\right) \downarrow \varphi(a)$.

(5.) For any net $\left(a_{\alpha}\right)_{\alpha \in \Lambda}$ of elements of $E_{1}$ and $a \in E_{1}$, $a_{\alpha} \stackrel{(o)_{1}}{\longrightarrow} a$ if and only if $\varphi\left(a_{\alpha}\right) \stackrel{(o)_{2}}{\longrightarrow} \varphi(a)$.

(6.) For subsets and nets of elements of $E_{1}$ and $E_{2}$ the following statements are satisfied:

- For all $F \subseteq E_{1}, F$ is $\tau_{0}^{E_{1}}$-closed if and only if $\varphi(F)$ is $\tau_{0}^{E_{2}}$-closed.

- For all $U \subseteq E_{1}, U$ is $\tau_{0}^{E_{1}}$-open if and only if $\varphi(U)$ is $\tau_{0}^{E_{2}}$-open.

- For any net $\left(a_{\alpha}\right)_{\alpha \in \Lambda}$ of elements of $E_{1}$ and $a \in E_{1}$, $a_{\alpha} \stackrel{\tau_{0}^{E_{1}}}{\longrightarrow} a$ if and only if $\varphi\left(a_{\alpha}\right) \stackrel{\tau_{0}^{E_{2}}}{\longrightarrow} \varphi(a)$. 
Proof. (1.) Assume that $a, b \in E_{1}$. If $a \leq_{1} b$. From Lemma 2.8 we have that $\varphi(a) \leq_{2} \varphi(b)$. Conversely, let $\varphi(a) \leq_{2} \varphi(b)$.Then again by Lemma 2.8 applied to $\varphi^{-1}$ we get $a=\varphi^{-1}(\varphi(a)) \leq_{1} \varphi^{-1}(\varphi(b))=b$.

(2.) Assume that $S \subseteq E_{1}$ such that $\bigvee_{E_{1}} S$ exists. Let us put $a=\bigvee_{E_{1}} S$. Then, for all $s \in S, s \leq a$ and we get from Lemma 2.8 that $\varphi(s) \leq_{1} \varphi(a)$. Hence $\varphi(a)$ is an upper bound of $\varphi(s)$ for all $s \in S$. Let $d=\varphi(c) \in E_{2}, c \in E_{1}$ be an upper bound of $\varphi(s)$ for all $s \in S$. Then $c \in E_{1}$ is an upper bound of $s$ for all $s \in S$ by Part 1 . This yields that $a \leq c$. Therefore by Lemma 2.8 we get $\varphi(a) \leq \varphi(c)=d$, i.e. $\bigvee_{E_{2}} \varphi(S)=\varphi\left(\bigvee_{E_{1}} S\right)$.

The converse implication follows by the same considerations as were applied above to $\varphi^{-1}$ and the assumption that $\bigvee_{E_{2}} \varphi(S)$ exists.

(3.) Assume $\alpha \leq \beta, \alpha, \beta \in \Lambda$. Then $a_{\alpha} \leq_{1} a_{\beta}$ and by Lemma 2.8 we obtain that $\varphi\left(a_{\alpha}\right) \leq_{2} \varphi\left(a_{\beta}\right)$. It follows that $\left(\varphi\left(a_{\alpha}\right)\right)_{\alpha \in \Lambda}$ is an increasingly directed net. Assume now that $a_{\alpha} \uparrow a$. Then by (2.) we obtain $\varphi\left(a_{\alpha}\right) \uparrow \varphi(a)$.

The converse implication follows by the same considerations as were applied above to $\varphi^{-1}$ and $\varphi\left(a_{\alpha}\right) \uparrow \varphi(a)$.

(4.) It follows by the considerations dual to them in (3.).

(5.) Assume first that $a_{\alpha} \stackrel{(o)_{1}}{\longrightarrow} a$. Hence there are nets $\left(u_{\alpha}\right)_{\alpha \in \Lambda}$ and $\left(v_{\alpha}\right)_{\alpha \in \Lambda}$ of elements of $E_{1}$ such that $a \uparrow u_{\alpha} \leq a_{\alpha} \leq v_{\alpha} \downarrow a$. From Lemma 2.8 and (3.) and (4.) we obtain nets $\left(\varphi\left(u_{\alpha}\right)\right)_{\alpha \in \Lambda}$ and $\left(\varphi\left(v_{\alpha}\right)\right)_{\alpha \in \Lambda}$ of elements of $E_{2}$ such that $\varphi(a) \uparrow \varphi\left(u_{\alpha}\right) \leq \varphi\left(a_{\alpha}\right) \leq$ $\varphi\left(v_{\alpha}\right) \downarrow \varphi(a)$. It follows that $\varphi\left(a_{\alpha}\right) \stackrel{(o)_{2}}{\longrightarrow} \varphi(a)$.

The converse implication follows by the same considerations as above applied to $\varphi^{-1}$ and the net $\left(\varphi\left(a_{\alpha}\right)\right)_{\alpha \in \Lambda}$ of elements of $E_{2}$ and $\varphi(a) \in E_{2}$ such that $\varphi\left(a_{\alpha}\right) \stackrel{(o)_{2}}{\longrightarrow} \varphi(a)$.

(6.) Assume that $F \subseteq E_{1}$. Then $F$ is $\tau_{0}^{E_{1}}$-closed if and only if by Theorem 2.6 for every net $\left(a_{\alpha}\right)_{\alpha \in \Lambda}$ of elements of $E_{1}$ it holds $\left(a_{\alpha} \in F, \alpha \in \Lambda, a_{\alpha} \stackrel{(o)_{1}}{\longrightarrow}\right.$ $a) \Rightarrow a \in F$ if and only if by (5.) for every net $\left(\varphi\left(a_{\alpha}\right)\right)_{\alpha \in \Lambda}$ of elements of $E_{2}$ it holds $\left(\varphi\left(a_{\alpha}\right) \in\right.$ $\left.\varphi(F), \alpha \in \Lambda, \varphi\left(a_{\alpha}\right) \stackrel{(o)_{2}}{\longrightarrow} \varphi(a)\right) \Rightarrow \varphi(a) \in \varphi(F)$ if and only if for every net $\left(b_{\alpha}\right)_{\alpha \in \Lambda}$ of elements of $E_{2}$ it holds $\left(b_{\alpha} \in \varphi(F), \alpha \in \Lambda, b_{\alpha} \stackrel{(o)_{2}}{\longrightarrow} b\right) \Rightarrow b \in \varphi(F)$ if and only if by Theorem 2.6 $\varphi(F)$ is $\tau_{0}^{E_{2}}$-closed. Now, let us assume that $U \subseteq E_{1}$. Then $U$ is $\tau_{0}^{E_{1}}$ open if and only if $F=E_{1} \backslash U$ is $\tau_{0}^{E_{1}}$-closed if and only if $\varphi(F)=\varphi\left(E_{1} \backslash U\right)=\varphi\left(E_{1}\right) \backslash \varphi(U)=$ $E_{2} \backslash \varphi(U)$ is $\tau_{0}^{E_{2}}$-closed if and only if $\varphi(U)$ is $\tau_{0}^{E_{2}}$ open.

In what remains, we will assume that we have a net $\left(a_{\alpha}\right)_{\alpha \in \Lambda}$ of elements of $E_{1}$ and $a \in E_{1}, a_{\alpha} \stackrel{\tau_{0}^{E_{1}}}{\longrightarrow}$ $a$. Let us check that $\varphi\left(a_{\alpha}\right) \stackrel{\tau_{0}^{E_{2}}}{\longrightarrow} \varphi(a)$. Assume that we have a $\tau_{0}^{E_{2}}$-open set $V \subseteq E_{2}$ such that $\varphi(a) \in V$. Since $V=\varphi(U)$ and $U=\varphi^{-1}(V)$ for some $\tau_{0}^{E_{1}}$-open subset $U \subseteq E_{1}$ we get that $a \in$ $U$. Hence there is an index $\alpha_{0} \in \Lambda$ such that $a_{\alpha} \in U$ for all $\alpha \geq \alpha_{0}$. It follows that $\varphi\left(a_{\alpha}\right) \in$ $\varphi(U)=V$ for all $\alpha \geq \alpha_{0}$. Therefore $\varphi\left(a_{\alpha}\right) \stackrel{\tau_{0}^{E_{2}}}{\longrightarrow}$ $\varphi(a)$. The converse implication goes the same way.

Recall that Theorem 3.1 can be stated and proved entirely for posets. Theorem 3.2 has to be stated and proved for effect algebras. Effect algebras are suitable algebraic structures to be carriers of states or probability measures ( $\sigma$-additive states) also in cases when events may be unsharp or some pairs of events are noncompatible.

Theorem 3.2. Let $\left(E_{1} ; \oplus_{1}, 0_{1}, 1_{1}\right)$ and $\left(E_{2} ; \oplus_{2}\right.$, $\left.0_{2}, 1_{2}\right)$ be effect algebras and let $\varphi: E_{1} \rightarrow E_{2}$ be an isomorphism of effect algebras. Then, for any mapping $\omega: E_{2} \rightarrow[0,1]$,

(1.) $\omega$ is a state on $E_{2}$ if and only if $\omega \circ \varphi$ is a state on $E_{1}$.

(2.) $\omega$ is an (o)-continuous state on $E_{2}$ if and only if $\omega \circ \varphi$ is an (o)-continuous state on $E_{1}$.

(3.) $\omega$ is a $\sigma$-additive state on $E_{2}$ if and only if $\omega \circ \varphi$ is a $\sigma$-additive state on $E_{1}$.

(4.) $\omega$ is a completely additive state on $E_{2}$ if and only if $\omega \circ \varphi$ is a completely additive state on $E_{1}$.

Proof. (1.) Let $\omega$ be a state on $E_{2}$. Then the composition $\omega \circ \varphi$ is a morphism from $E_{1}$ to $[0,1]$, hence a state. Conversely, let $\omega \circ \varphi$ be a state on $E_{1}$. Then $\omega=(\omega \circ \varphi) \circ \varphi^{-1}$ is a morphism from $E_{2}$ to $[0,1]$.

(2.) Let $\omega$ be an $(o)$-continuous state on $E_{2}$. Assume that $\left(a_{\alpha}\right)_{\alpha \in \Lambda}$ is an increasingly directed net of elements of $E_{1}$ and that $a \in E_{1}$ such that $a_{\alpha} \uparrow a$. From Theorem 3.1 we obtain that $\varphi\left(a_{\alpha}\right) \uparrow \varphi(a)$ in $E_{2}$. Since $\omega$ is $(o)$-continuous we have that $\omega\left(\varphi\left(a_{\alpha}\right)\right) \uparrow \omega(\varphi(a))$. Hence, by (1.), $\omega \circ \varphi$ is an (o)-continuous state on $E_{1}$.

The converse implication follows by the same considerations as above applied to $\varphi^{-1}$ and the $(o)$ continuous state $\omega \circ \varphi$ on $E_{1}$.

(3.) It follows by literally the same considerations as in (2.) applied to any countable increasingly directed net.

(4.) Let $\omega$ be a completely additive state on $E_{2}$. Assume that $\left(x_{\kappa}\right)_{\kappa \in H}$ is an orthogonal system of not necessarily different elements of $E_{1}$ such that $\bigoplus_{E_{1}}\left\{x_{\kappa} \mid \kappa \in H\right\}$ exists. Then by Lemma 2.8 we get that $\left(\varphi\left(x_{\kappa}\right)\right)_{\kappa \in H}$ is an orthogonal system in $E_{2}$ and by Theorem 3.1 we obtain that $\varphi\left(\bigoplus_{E_{1}}\left\{x_{\kappa} \mid \kappa \in H\right\}\right)=\bigoplus_{E_{2}}\left\{\varphi\left(x_{\kappa}\right) \mid \kappa \in H\right\}$. Since $\omega$ is a completely additive state on $E_{2}$ we 
have that

$$
\begin{aligned}
& (\omega \circ \varphi)\left(\bigoplus_{E_{1}}\left\{x_{\kappa} \mid \kappa \in H\right\}\right) \\
& =\omega\left(\bigoplus_{E_{2}}\left\{\varphi\left(x_{\kappa}\right) \mid \kappa \in H\right\}\right) \\
& =\sum\left\{\omega\left(\varphi\left(x_{\kappa}\right)\right) \mid \kappa \in H\right\} \\
& =\sup \left\{\sum\left\{(\omega \circ \varphi)\left(x_{\kappa}\right) \mid \kappa \in F\right\} \mid \begin{array}{l}
F \subseteq H, \\
F \text { finite set }\} .
\end{array}\right.
\end{aligned}
$$

The converse implication follows by the same considerations as above applied to $\varphi^{-1}$.

\section{SOME PROPERTIES OF OPERATOR}

\section{EFFECT ALGEBRAS THAT NEED NOT BE PRESERVED BY EFFECT}

\section{ALGEBRAIC ISOMORPHISMS}

We see, in Section 3, that isomorphism of effect algebras preserves those properties of effect algebras which depend only on the $\oplus$-operation or on the partial order that is derived from $\oplus$. On the other hand, there are properties of effect algebras for which the preservation of the $\oplus$-operation by isomorphisms is not substantional. For operator effect algebras it is, e.g., boundedness or self-adjointness of operators (elements of operator effect algebras).

Definition 4.1. [4] A sequential effect algebra is a partial algebra $(E ; \circ, \oplus, 0,1)$ such that $(E ; \oplus, 0,1)$ is an effect algebra and $\circ$ is another binary operation (called a sequential product) defined on $E$ satisfying:

(SEA1) The map $b \mapsto a \circ b$ is additive for each $a \in E$, that is, if $b \perp c$, then $a \circ b \perp a \circ c$ and $a \circ(b \oplus c)=a \circ b \oplus a \circ c$.

(SEA2) $1 \circ a=a$ for each $a \in E$.

(SEA3) If $a \circ b=0$, then $a \circ b=b \circ a$.

(SEA4) If $a \circ b=b \circ a$, then $a \circ b^{\prime}=b^{\prime} \circ a$ and $a \circ(b \circ c)=(a \circ b) \circ c$ for each $c \in E$.

(SEA5) If $c \circ a=a \circ c$ and $c \circ b=b \circ c$, then $c \circ(a \circ b)=(a \circ b) \circ c$ and $c \circ(a \oplus b)=(a \oplus b) \circ c$ whenever $a \perp b$.

Assume that $\left(E_{1} ; \circ_{1}, \oplus_{1}, 0_{1}, 1_{1}\right)$ and $\left(E_{2} ; \circ_{2}, \oplus_{2}, 0_{2}, 1_{2}\right)$ are sequential effect algebras. A mapping $\varphi: E_{1} \rightarrow E_{2}$ is called a sequential effect algebraic morphism if $\varphi$ is a morphism of the effect algebra $E_{1}$ into the effect algebra $E_{2}$ and, for all $a, b \in E_{1}, \varphi\left(a \circ_{1} b\right)=$ $\varphi(a) \circ_{2} \varphi(b)$.

In what follows we will assume that $\mathcal{H}$ is an infinitedimensional complex Hilbert space, i.e., a linear space with inner product $(\cdot, \cdot)$ which is complete in the induced metric. The term dimension of $\mathcal{H}$ is defined as the cardinality of any orthonormal basis of $\mathcal{H}$ (see [1]).

Moreover, we will assume that all considered linear operators $A$ (i.e. linear maps $A: D(A) \rightarrow \mathcal{H}$ ) have a domain $D(A)$ that is a linear subspace dense in
$\mathcal{H}$ with respect to the metric topology induced by the inner product on $\mathcal{H}$ (i.e., $\overline{D(A)}=\mathcal{H}$ ). Recall that a linear operator $A$ is called positive (denoted by $A \geq 0)$ iff $(x, A x) \geq 0$ for all $x \in D(A)$, hence $A$ is also symmetric, meaning that $(y, A x)=(A y, x)$ for all $x, y \in D(A)$ (see [1] for more details).

Recall that $A: D(A) \rightarrow \mathcal{H}$ is called a bounded operator if there exists a real constant $C \geq 0$ such that $\|A x\| \leq C\|x\|$ for all $x \in D(A)$.

Gudder 4] showed that, for any standard Hilbert space effect algebra $\mathcal{E}(\mathcal{H})$ on a complex Hilbert space $\mathcal{H}$, there is a binary operation $\circ$ defined by $B \circ C=$ $B^{\frac{1}{2}} C B^{\frac{1}{2}}$ for all $B, C \in \mathcal{E}(\mathcal{H})$ such that it satisfies conditions (SEA1)-(SEA5), and so it is a sequential product of $\mathcal{E}(\mathcal{H})$. Liu Weihua and Wu Junde in [10, Theorem 4.3] proved that there is a binary operation ${ }^{\circ}$ on $\mathcal{E}(\mathcal{H})$ such that it satisfies conditions (SEA1)(SEA5) and $\circ_{i} \neq \circ$. This yields the following.

Theorem 4.2. Let $\mathcal{H}$ be a complex Hilbert space. Then there are sequential operator effect algebras $(\mathcal{E}(\mathcal{H}) ; \circ, \oplus, 0,1)$ and $\left(\mathcal{E}(\mathcal{H}) ; \circ_{i}, \oplus, 0,1\right)$ that are isomorphic as effect algebras but the respective effect algebraic isomorphism does not preserve the sequential product.

Proof. Evidently, $\operatorname{id}_{\mathcal{E}(\mathcal{H})}$ is an effect algebraic isomorphism. From [10, Theorem 4.3] we know that there are $A, B \in \mathcal{E}(\mathcal{H})$ such that $A \circ B \neq A \circ_{i} B$. Hence $\operatorname{id}_{\mathcal{E}(\mathcal{H})}(A \circ B)=A \circ B \neq A \circ_{i} B=\operatorname{id}_{\mathcal{E}(\mathcal{H})}(A) \circ_{i}$ $\operatorname{id}_{\mathcal{E}(\mathcal{H})}(B)$.

Let $\mathcal{V}(\mathcal{H})$ be the set of all positive linear operators densely defined in an infinite-dimensional complex Hilbert space $\mathcal{H}$ and the domain $D(B)=\mathcal{H}$ for every bounded operator $B$. To every such linear operator with $\overline{D(A)}=\mathcal{H}$ there exists the adjoint operator $A^{*}$ of $A$ such that $D\left(A^{*}\right)=\left\{y \in \mathcal{H} \mid\right.$ there exists $y^{*} \in \mathcal{H}$ such that $\left(y^{*}, x\right)=(y, A x)$ for every $\left.x \in D(A)\right\}$ and $A^{*} y=y^{*}$ for every $y \in D\left(A^{*}\right)$. If $A^{*}=A$ then $A$ is called self-adjoint.

An operator $A: D(A) \rightarrow \mathcal{H}$ is called closed if for every sequence $\left(x_{n}\right)_{n \in \mathbb{N}}, x_{n} \in D(A)$, such that $x_{n} \rightarrow x \in \mathcal{H}$ and $A x_{n} \rightarrow y \in \mathcal{H}$ as $n \rightarrow \infty$ one has $x \in D(A)$ and $A x=y$. Since every $A \in \mathcal{V}(\mathcal{H})$ is symmetric there exists a closed operator $\bar{A}$ such that $A \subset \bar{A}$ and $\bar{A} \subset B$ for every closed operator extending $A$. Moreover $\bar{A}$ is again symmetric and it is called the closure of $A$. A symmetric operator $A$ is called essentially self-adjoint if $(\bar{A})^{*}=\bar{A}$ and then $\bar{A}$ is a unique self-adjoint extension of $A$ (see [1, p. 96]). Finally, recall that every $A \in \mathcal{V}(\mathcal{H})$ has a positive self-adjoint extension $\hat{A}$ called Friedrichs' extension of $A$ (see, e.g., [16]). Moreover, $\hat{A}$ extends all symmetric extensions $A^{\prime}$ of $A$. It was shown in [15, Theorem 1] that, for any infinite-dimensional complex Hilbert space $\mathcal{H}$, there are positive unbounded $A$ and $B$ such that $A$ is not essentially self-adjoint and $B$ is not closed. 
Furthermore, let $\mathcal{V}(\mathcal{H})$ be equipped with the partial sum $\oplus$ such that for any $A, B \in \mathcal{V}(\mathcal{H})$ the sum $A \oplus B$ is defined iff either one of $A, B$ is bounded or $D(A)=$ $D(B)$. Then we set $A \oplus B=A+B$ (the usual operator sum). In [17] it was proved that $(\mathcal{V}(\mathcal{H}) ; \oplus, 0)$ is a generalized effect algebra.

Theorem 4.3 ([17, Theorem 2], [18, Theorem 7]). For every infinite-dimensional complex Hilbert space $\mathcal{H}$ and every $Q \in \mathcal{V}(\mathcal{H}), Q \neq 0$ it holds:

(1.) The interval $\left([0, Q]_{\mathcal{V}(\mathcal{H})} ; \oplus_{Q}, 0, Q\right)$ where $A \oplus_{Q}$ $B=A+B$ iff $A+B \leq Q$, for any $A, B \in$ $[0, Q]_{\mathcal{V}(\mathcal{H})}$, is an effect algebra and $\mathcal{M}_{Q}=$ $\left\{\omega_{x} \mid x \in D(Q),(x, Q x)>0\right\}$ is an ordering set of states on $[0, Q]_{\mathcal{V}(\mathcal{H})}$; here the mapping $\omega_{x}:[0, Q]_{\mathcal{V}(\mathcal{H})} \rightarrow[0,1] \subseteq \mathbb{R}$ is defined for every $A \in[0, Q]_{\mathcal{V}(\mathcal{H})}$ by $\omega_{x}(A)=$ $\frac{(x, A x)}{(x, Q x)}$.

(2.) The effect algebra $\left([0, Q]_{\mathcal{V}(\mathcal{H})} ; \oplus_{Q}, 0, Q\right)$ can be embedded into the standard Hilbert effect algebra $\mathcal{E}\left(l_{2}\left(\mathcal{M}_{Q}\right)\right)$. We denote the respective embedding by $\varphi_{Q} \cdot$

Therefore we obtain the following theorem that boundedness (self-adjointness, closedness, essential self-adjointness, Friedrichs' extension) of operators need not be preserved by effect algebraic isomorphisms.

Theorem 4.4. For every infinite-dimensional complex Hilbert space $\mathcal{H}$ and every $Q \in \mathcal{V}(\mathcal{H}), Q \neq 0$ unbounded (unbounded and non self-adjoint, unbounded and non closed, unbounded and non essentially self-adjoint, unbounded and with $\hat{Q} \neq Q$ respectively) we have an effect algebraic isomorphism $\varphi_{Q}^{-1}: \varphi_{Q}\left([0, Q]_{\mathcal{V}(\mathcal{H})}\right) \rightarrow[0, Q]_{\mathcal{V}(\mathcal{H})}$ such that $\varphi_{Q}^{-1}$ does not preserve bounded operators (self-adjoint operators, closed operators, non essentially self-adjoint operators, Friedrichs' extension, respectively).

Proof. Clearly, $\varphi_{Q}(Q)=I_{l_{2}\left(\mathcal{M}_{Q}\right)} \in \varphi_{Q}([0, Q] \mathcal{V}(\mathcal{H}))$. Note that $I_{l_{2}\left(\mathcal{M}_{Q}\right)}$ is bounded and positive. It follows that it is also self-adjoint, closed, essentially self-adjoint and it coincides with its Friedrichs' extension. Hence $\varphi_{Q}^{-1}\left(I_{l_{2}\left(\mathcal{M}_{Q}\right)}\right)=Q, I_{l_{2}\left(\mathcal{M}_{Q}\right)}$ is bounded (self-adjoint, closed, essentially self-adjoint and it coincides with its Friedrichs' extension respectively) in $l_{2}\left(\mathcal{M}_{Q}\right)$ and $Q$ is unbounded (unbounded and non self-adjoint, unbounded and non closed, unbounded and non essentially self-adjoint, unbounded and with $\hat{Q} \neq Q$, respectively).

\section{ACKNOWLEDGEMENTS}

The first author acknowledges support from ESF Project CZ.1.07/2.3.00/20.0051 Algebraic methods in Quantum Logic of the Masaryk University. The second author was supported by grant VEGA $1 / 0297 / 11$ of the Ministry of Education of the Slovak Republic, and by project APVV0178/11

\section{REFERENCES}

[1] J. Blank, P. Exner, M. Havlíček, Hilbert Space Operators in Quantum Physics (Second edition), Springer, 2008.

[2] Dvurečenskij A., Pulmannová S., New trends in quantum structures, Kluwer, Dordrecht, the Netherlands, 2000.

[3] Foulis D.J., Bennett M.K., Effect algebras and unsharp quantum logics, Foundations of Physics 24 (1994), 1331-1352.

[4] Gudder S., Greechie R., Sequential products on effect algebras, Reports of Mathematical Physics, 49 (2002), 87-111.

[5] Hedlíková J., Pulmannová S., Generalized difference posets and orthoalgebras, Acta Math. Univ. Comenianae LXV (1996), 247-279.

[6] Kalmbach G., Riečanová Z., An axiomatization for abelian relative inverses, Demonstratio Math. 27 (1994), 769-780.

[7] Kirchheimová H., Some remarks on (o)-converfengence, Proc. of the First Winter School of Measure Theory, Liptovský Ján (1990), 110-113.

[8] Kirchheimová H., Riečanová Z., Note on order convergence and order topology, Appendix B, in Riečan, B., Neubrunn, T., Measure, Integral and Order, Ister Science (Bratislava) and Kluwer Academic Publishers (Dordrecht-Boston-London), 1997.

[9] Kôpka F., Chovanec F., D-posets, Math. Slovaca 44 (1994), 21-34.

[10] Liu, W. H., Wu, J. D., The uniqueness problem of sequence product on operator effect algebra $\mathcal{E}(\mathcal{H}), J$. Phys. A: Math. Theor. 42 (2009).

[11] Mosná K., Paseka J., Riečanová Z., Order convergence, order and interval topologies on posets and lattice effect algebras, In UNCERTAINTY 2008, Bratislava, Slovak Republic: Slovak University of Technology in Bratislava, Publishing House of STU, (2008), 45-62,

[12] Olejček V., Order convergence and order topology on a poset, Int. J. Theor. Physics 38 (1999), 557-561.

[13] Olejček V., The Order Topology on a Lattice and Its MacNeille Completion, Int. J. Theor. Physics, 39 (2000), 801-803.

[14] Paseka J., Riečanová Z., The inheritance of BDE-property in sharply dominating lattice effect algebras and (o)-continuous states, Soft Computing, 15 (2011), 543-555.

[15] Paseka J., Riečanová Z., Considerable Sets of Linear Operators in Hilbert Spaces as Operator Generalized Effect Algebras, Foundations of Physics, 41 (2011), 1634-1647.

[16] Reed M., Simon B., Methods of Modern Mathematical Physics II, Fourier Analysis, Self-Adjointness, Academic Press New York, San Francisco, London, 1975.

[17] Riečanová Z., Zajac M., Pulmannová S., Effect algebras of positive linear operators densely defined on Hilbert spaces, Reports of Mathematical Physics, 68 (2011), 261-270.

[18] Riečanová Z., Zajac M., Hilbert space effect-representations of effect algebras, Reports of Mathematical Physics, 70 (2012), 283-290. 